OPEN ACCESS

Edited by:

Ruey-Meei Wu,

National Taiwan University, Taiwan

Reviewed by:

Santiago Perez-Lloret, National Council for Scientific and

Technical Research (CONICET),

Argentina

Yih-Ru Wu

Chang Gung Memorial Hospital,

Taiwan

*Correspondence:

Jin Wang

wangjin72@126.com

${ }^{\dagger}$ These authors have contributed equally to this work

Specialty section:

This article was submitted to Movement Disorders,

a section of the journal

Frontiers in Neurology

Received: 16 December 2018 Accepted: 29 March 2019

Published: 17 April 2019

Citation:

Qiu X, Xiao Y, WU J, Gan L, Huang Y and Wang J (2019) C-Reactive Protein and Risk of Parkinson's Disease: A

Systematic Review and

Meta-Analysis. Front. Neurol. 10:384.

doi: 10.3389/fneur.2019.00384

\section{C-Reactive Protein and Risk of Parkinson's Disease: A Systematic Review and Meta-Analysis}

\author{
Xiaohui Qiu', Yousheng Xiao ${ }^{\dagger}$, Jingjing Wu, Lu Gan, Yanning Huang and Jin Wang* \\ Department of Neurology, The First Affiliated Hospital of Guangxi Medical University, Nanning, China
}

Background: C-reactive protein (CRP) has been identified as a common inflammation-related cytokine. Although publications indicate that CRP is associated with the pathogenesis of neurological disorders and deemed to be a "risk factor" for Parkinson's disease (PD), the evidence exists still indefinitely. Here, we performed a systematic review with meta-analysis synthesizing all the eligible studies on serum, plasma, and cerebrospinal fluid (CSF) CRP levels and PD risk to investigate the potential relevance.

Methods: A systematical search up to October 2018 was performed via PubMed, Embase, Science Direct, ISI Web of Science as well as three Chinese medical databases: China National Knowledge Infrastructure database (CNKI), VIP database and WanFang database. Risk was assessed by standardized mean difference (SMD) with 95\% confidence interval $(\mathrm{Cl})$ to investigate the involvement of CRP levels in PD patients.

Results: Twenty-three eligible case-control studies involving 4,598 individuals (2,646 PD patients and 1,932 healthy controls) were incorporated into this meta-analysis. Results have indicated significant increase of CRP levels in PD subjects when compared with control groups in serum (SMD $=1.115,95 \% \mathrm{Cl} 0.619-1.61, P<0.001)$, CSF $(\mathrm{SMD}=$ $1.127,95 \% \mathrm{Cl} 0.133-2.120, P=0.026)$ as well as whole blood $(\mathrm{SMD}=1.071,95 \% \mathrm{Cl}$ $0.715-1.426, P<0.001)$.

Conclusions: This meta-analysis revealed that PD is associated with an increase of CRP levels. CRP might be a risk factor for PD or PD leads to an inflammatory response.

Keywords: Parkinson's disease, C-reactive protein, risk factor, meta-analysis, inflammation

\section{INTRODUCTION}

Parkinson's disease (PD) is one of the most complex neuro-degenerative disorders next to Alzheimer's disease. It is characterized by bradykinesia, tremor, rigidity, abnormal postural, and gait (1). PD has been recognized by the selective loss of dopaminergic neurons within the substantia nigra pars compacta, whereas the exact etiology remains elusive (2). Previously, multiple inferences have reviewed the environmental and behavioral factors as the risk of developing $\mathrm{PD}$, such as high milk and dairy consumption, exposure to pesticides and other environmental chemicals, history of melanoma, usage of amphetamine or methamphetamine, and traumatic brain injury, etc (3). However, the last decades, testable hypotheses were build that microglia-mediated neuroinflammation may contribute to the pathogenesis of PD (4-7). Furthermore, epidemiological studies show that anti-inflammatory medications, especially nonsteroidal anti-inflammatory drugs 
have neuroprotective effects and greatly reduced the risk of PD, which forcefully corroborating the above mentioned hypothesis $(8-11)$.

C-reactive protein (CRP), a kind of acute-phase protein and regulated by pro-inflammatory cytokines, is the most studied bio-marker of systemic inflammation (12). Some researches suggest that elevated levels of CRP is intensely associated with inflammatory process (13). Studies have also demonstrated a link between CRP and chronic inflammatory and neurodegenerative diseases, such as cardiovascular disease, diabetes, stroke, and Alzheimer's disease, as well as PD (14). Up to now, some epidemiological studies have explored CRP levels and PD risk. However, results in the literature regarding CRP levels in PD patients are still contradictory. Some studies found a significantly increase of CRP levels in subjects suffering PD compared with healthy controls (15-17), while some reports did not identify a similar tendency $(18,19)$. Therefore, we performed a systematic review via an extensive and comprehensive search that focus on serum, plasma, blood and CSF CRP levels in PD patients to clarify the potential risk of CRP levels in PD.

\section{MATERIALS AND METHODS}

\section{Search Strategy}

This meta-analysis was performed in line with the preferred reporting items for systematic reviews and meta-analyses (PRISMA) statement (20). A systematic review via PubMed, Embase, Science Direct, ISI Web of Science, and three Chinese medical databases: China National Knowledge Infrastructure database, VIP database, and WanFang database was searched since published from inception till October 2018. Search strategies included a combination of the following key words: ("C-reactive protein" $\mathrm{OR}$ "C reactive protein" OR CRP) and ("Parkinson Disease" OR "Parkinson's Disease" OR "Parkinsonism" OR PD). Both English and Chinese articles were enrolled. In addition, potential reviews and meta-analyses were examined manually to identify any additional related article that might be suitable for this review.

\section{Inclusion and Exclusion Criteria}

Two investigators (Qiu X, Xiao Y) independently identified and selected studies based on the Participants, Intervention, Comparison, Outcomes, and Study design (PICOS) framework (Table 1). Regarding to the reduplicative patient population, only the latest or most complete study was recruited.

\section{Data Extraction}

Data was extracted from enrolled articles independently by two investigators (Qiu X, Xiao Y). We collected the following data: (1) general information: first author, publication year, country of the population and study subjects; (2) patient characteristics: sample size, age, gender, clinical setting, adjusted variables for controls; (3) CRP assay type; (4) CRP level. Inconsistencies between the two authors were resolved by consulting a third reviewer (Wang J), and a level of 95\% agreement was achieved. We contacted the investigators of the research for further information when required.

\section{Quality Assessment}

Quality of each study was evaluated on the basis of the NewcastleOttawa Scale (NOS) (39). There were three domains outlined in the NOS: "Selection," "Comparability," and "Outcome." NOS scores with nine points were adopted to determine the quality categories. High quality was defined with 7 scores or more, intermediate quality from 4 to 6 and inferior quality below 4 .

\section{Statistical Analysis}

The software STATA version 12.0 (StataCorp LP, College Station, TX, USA) was employed to perform this metaanalysis investigating the correlation of PD risk and CRP levels. Standardized mean difference (SMD) with 95\% confidence interval (CI) for continuous outcomes was used to measure the differences of CRP levels between PD patients and healthy controls. The value of SMD $<0.2$ implied a slight effect, indicating a low risk between CRP levels and PD, 0.5 indicated a moderate effect, and exceeding 0.8 suggested a significant effect (40). Heterogeneity among trials were quantified by $Q$ chi-square test and $I^{2}$ metric. Substantial heterogeneity exists when $I^{2}$ exceeding $50 \%$ and $P$-value $<0.5$ for the $Q$ test (41). A random- effects model was employed to merge the overall effect size if significant heterogeneity existed; otherwise, a fixed-effects model was preferred (42). Subgroup analyses were conducted to explore the possible causes of heterogeneity. Sensitivity analysis was utilized to value the influence of individual research on the overall effect estimate. Funnel plot was applied to visually inspect publication bias, along with Egger's test (43). $P$-values $<0.05$ was supposed to statistically significant.

\section{RESULTS}

\section{Studies Selection}

A flowchart describing the study selection process is displayed in Figure 1. A total of 2,173 citations were yielded based on the search strategies above. After duplicates were removed, the remaining 1,811 articles were then independently reviewed. When filtrating titles and abstracts, a majority of 1,648 papers were excluded given that they were out of the predetermined criteria. Finally, 23 studies fulfilling the inclusion criteria were included in this meta-analysis (15-19, 21-38).

\section{Study Characteristics}

The descriptive data of the 23 studies are outlined in Table 2. The date of publication was between 2009 and 2018. Sample size ranged from 40 to 800 subjects, with a number of 4,589 participants were included (a total of 2,646 cases and 1,932 controls). The mean age of PD patients ranged from 63.6 to 73.2 years. The disease duration of PD varied from 3 months to 9.8 years. Nineteen $(15,16,21-35,37,38)$ studies recruited participants in Asian, three $(18,19,36)$ in Europe and the remaining one (17) in South America. PD patients in nine researches $(15,17,18,24,26,32,35-37)$ were taking anti-PD drugs such as levodopa. Eleven trials (18, 19, 24-29, 31, 33, 34) enrolled the PD patients accompanied by dementia, anxiety or depression. Samples of 12 trials $(16,18,21,22,25-28,32-34,37)$ were from serum, three $(15,24,35)$ from plasma, four $(17,29-31)$ 


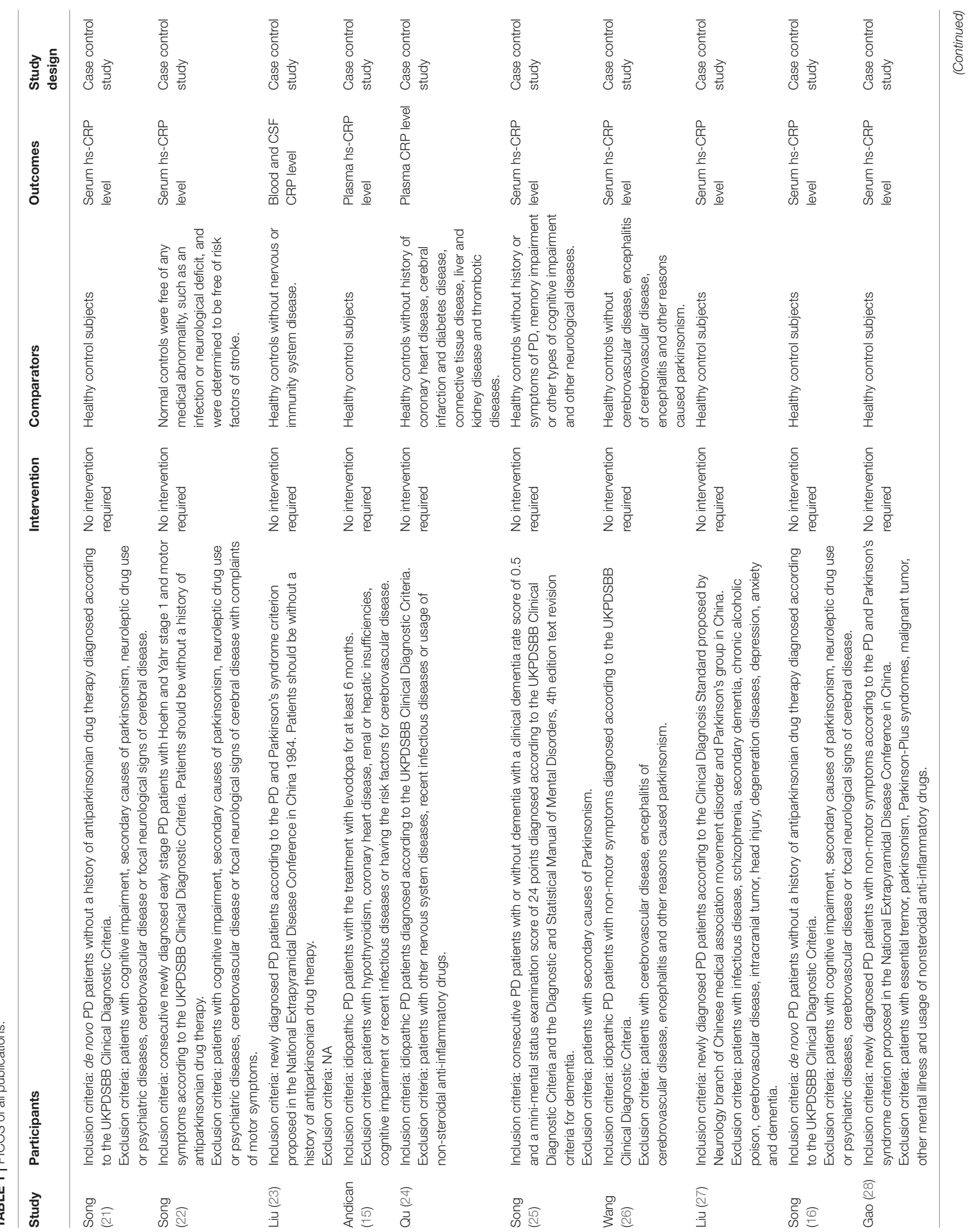




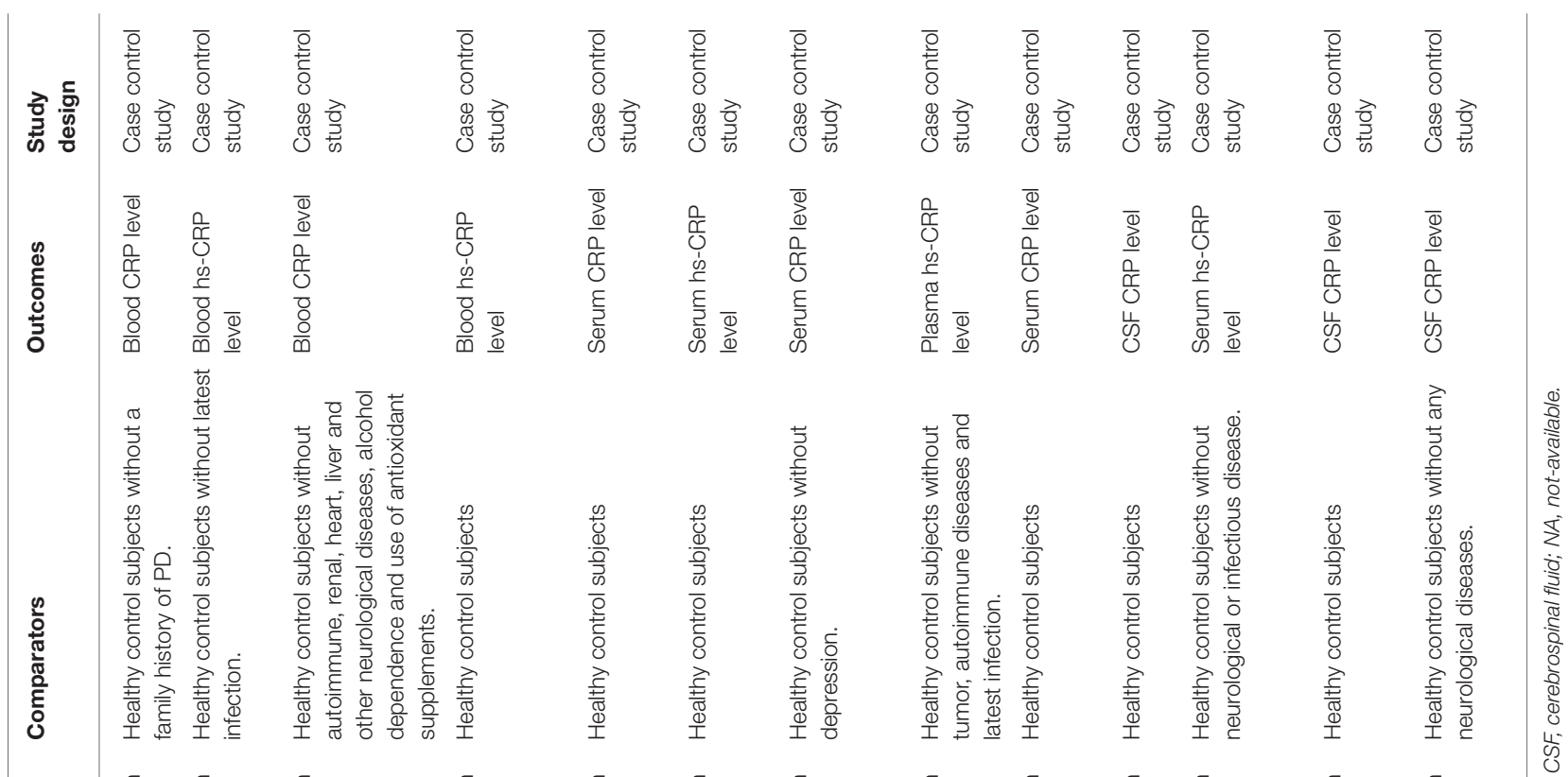

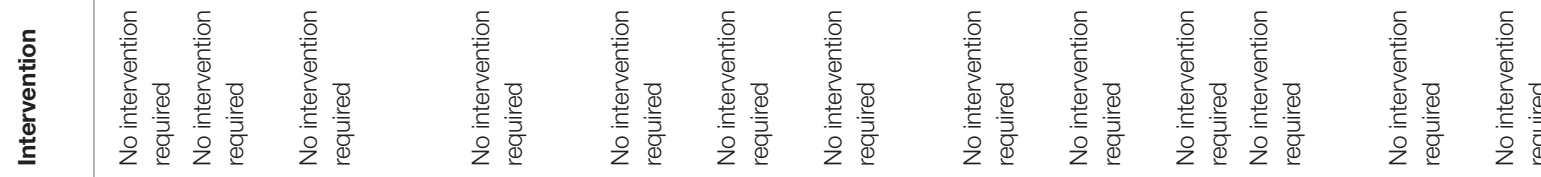

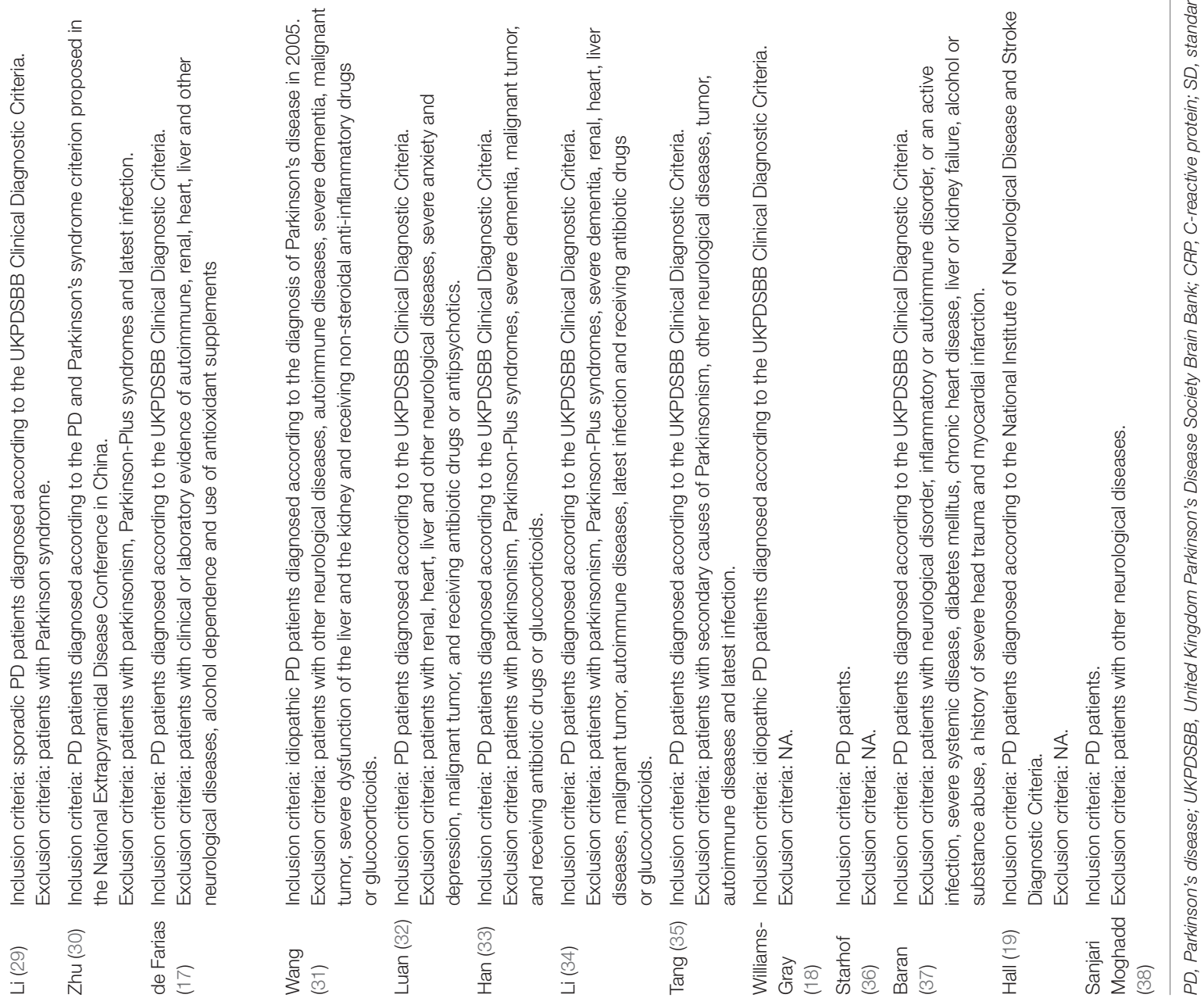




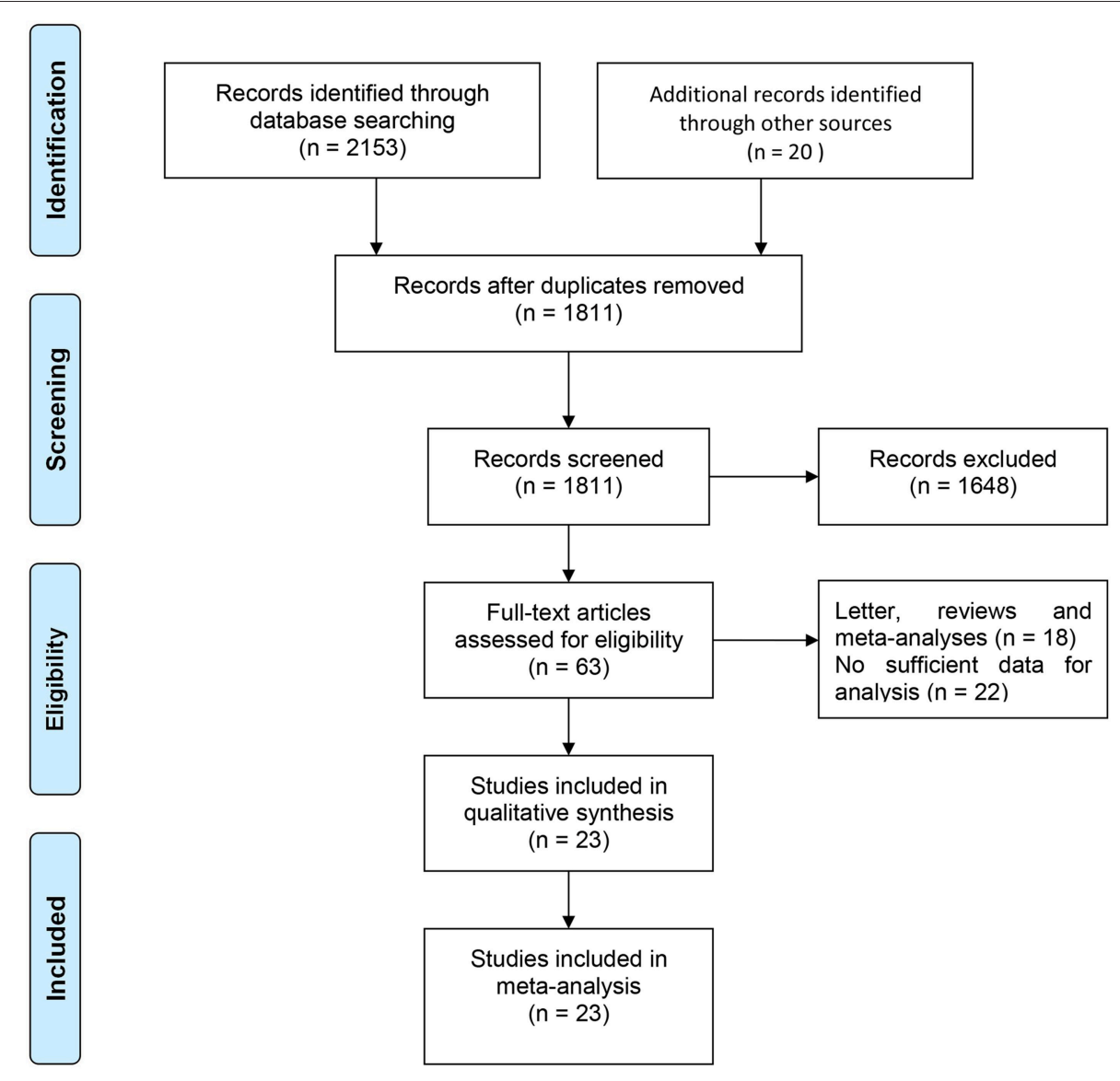

FIGURE 1 | Flowchart of the study identification, inclusion, and exclusion in the meta-analysis.

from the whole blood, three $(19,36,38)$ from CSF, and one (23) from both blood and CSF. Thirteen studies $(15,16,21,22,25-$ 28, 30, 31, 33, 35, 37) applied high-sensitivity CRP (hs-CRP) assay and $10(17-19,23,24,29,32,34,36,38)$ utilized standard CRP maker. The quality of the enrolled studies was assessed by means of Newcastle-Ottawa Scale. Ten (16, 17, 19, 21, 22, 24, 25, $29,37,38)$ out of 23 studies were assessed of high quality, 11 studies $(18,23,26-28,30,31,33-36)$ were assessed as medium quality, and the remaining two studies $(15,32)$ were evaluated as low quality.

\section{Meta-Analysis of CRP in Blood Levels}

Twenty studies (15-18, 21-35, 37) measured CRP levels in peripheral blood consisting of those samples derived from serum, plasma or whole blood of PD patients. A total of 4,127 subjects with 2,360 PD patients and 1,767 normal controls were included in this meta-analysis. Results showed that CRP levels in peripheral blood were significantly increased in PD patients compared to controls (SMD $=1.071,95 \% \mathrm{CI}$ : 0.715-1.426, $P<$ 0.001) (Figure 2), a random-effect model was used because of the heterogeneity $\left(I^{2}=96.2 \%, P<0.001\right)$.

Subgroup analyses were conducted according to CRP measurement manner. Results suggested that CRP levels were significantly increased in studies using hs-CRP as a measurement method (SMD = 1.150, 95\% CI: 0.655-1.634, $I^{2}=96.5 \%$ ). Sensitivity analysis was conducted to address the potential heterogeneity on account of the impact of enrolled studies on the overall effect size and its quality (Figure 3 ). The pooled SMD in CRP levels ranged from 0.877 (95\% CI: 0.583-1.171) to 1.134 (95\% CI: 0.755-1.514). Summary results were not influenced significantly by any one particular study, indicating strength and robustness of this meta-analysis. Asymmetry was identified in the funnel plot where four studies $(23,28,31,33)$ fell distantly outside of the predicted $95 \% \mathrm{CI}$, implying a potential publication bias should not be ignored (Figure 4). Egger's test $(P<0.001)$ also provided statistical evidence of publication bias.

\section{Meta-Analysis of CRP in Serum Levels}

Twelve studies (16, 18, 21, 22, 25-28, 32-34, 37) involving 2,553 participants that comparing the serum CRP levels in PD patients and healthy controls were analyzed. Nine studies $(16,21,22,25-$ $28,33,37)$ employed the hs-CRP maker to measure the serum CRP levels while the remaining three $(18,32,34)$ used CRP. In this meta-analysis, the serum CRP levels were significantly higher in PD patients compared to those healthy controls (SMD $=1.115,95 \%$ CI: $0.619-1.61, P<0.001$, Figure 5). Due to the 


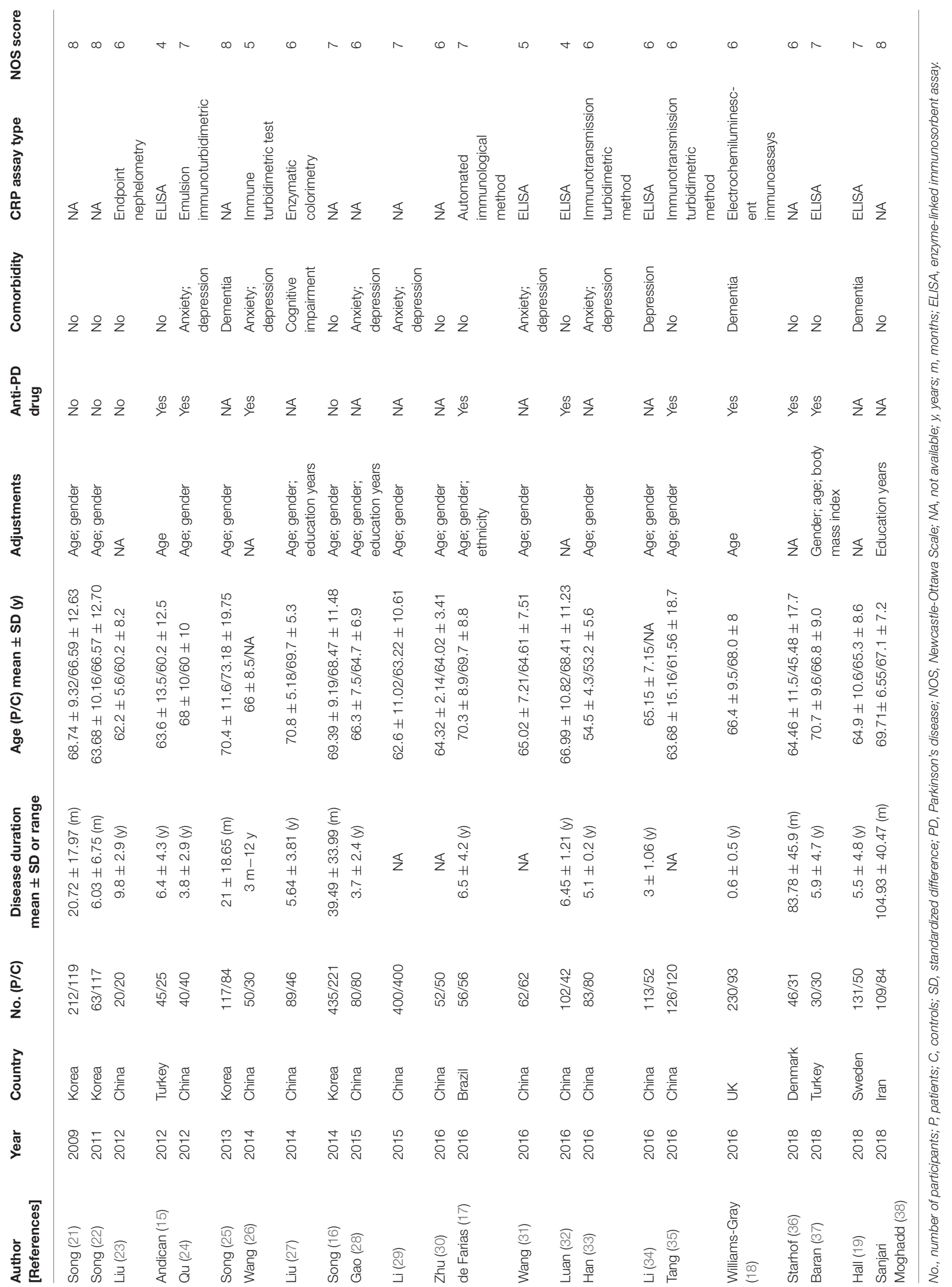




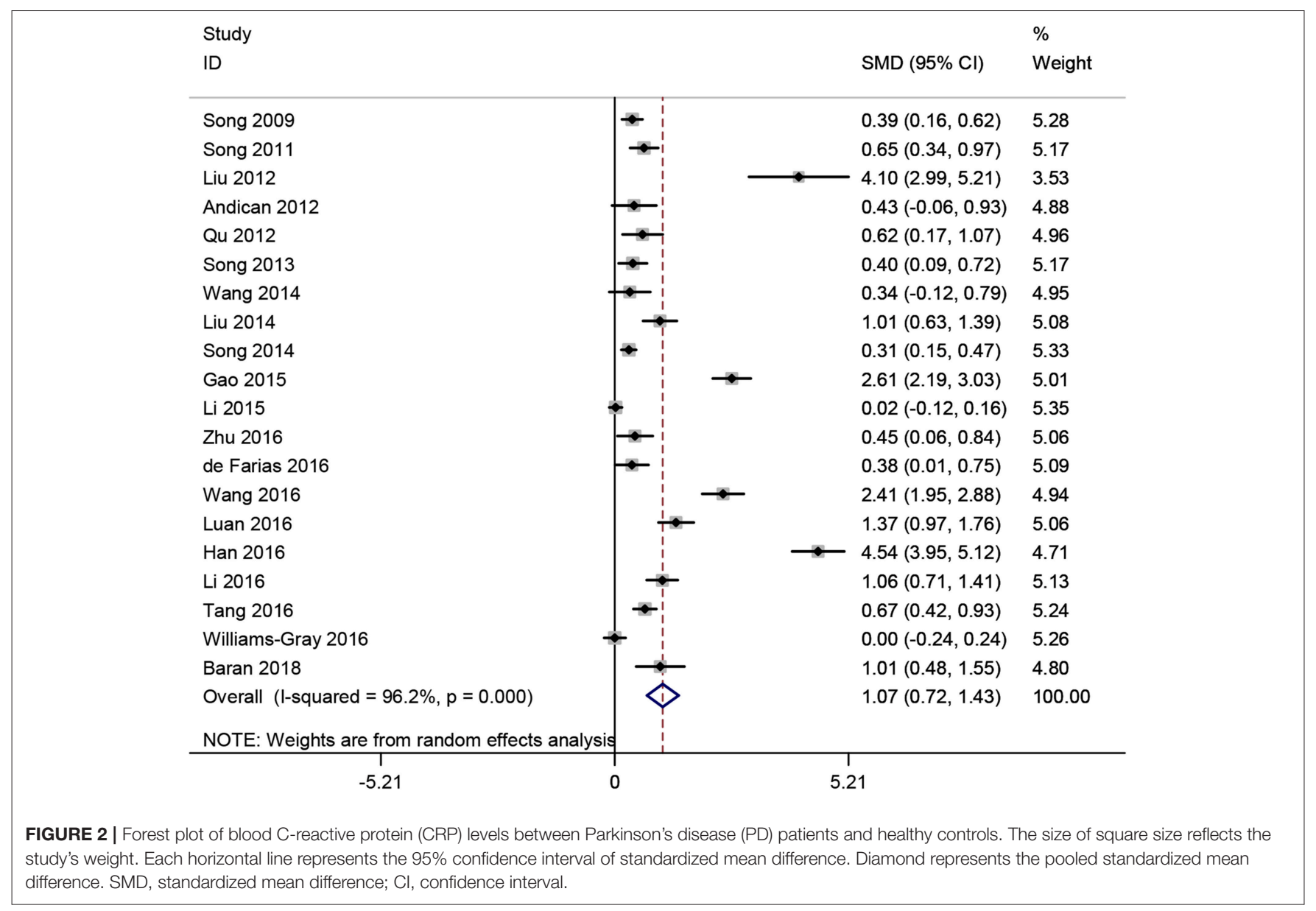

substantial heterogeneity detected $\left(I^{2}=96.7 \%, P<0.001\right)$, a random-effect model was employed $(44,45)$. Because of the similar clinical characteristics among these 12 studies, we did not carry out subgroup analyses. No individual study remarkably influenced the stability of the results in sensitivity analysis. However, Egger's linear regression test $(P=0.01)$ found the exist of publication bias.

\section{Meta-Analysis of CRP in CSF Levels}

CRP levels in CSF were analyzed in 491 participants from four studies $(19,23,36,38)$. Pooled analysis revealed that CRP levels in CSF were greatly increased in studies with PD patients compared to healthy controls (SMD $=1.127,95 \%$ CI: $0.133-$ $2.120, P<0.001$, Figure 6). Since obvious heterogeneity among those studies $\left(I^{2}=95.2 \%, P<0.001\right)$ were observed, a randomeffect model was utilized. We did not perform further subgroup analysis considering the limited number of included studies.

\section{DISCUSSION}

In recent years, the association between CRP and risk of PD has been widely studied. However, it is still not well understood. Herein, in this meta-analysis, we made a comprehensive comparison of the CRP levels in peripheral blood and CSF between PD patients and healthy controls. A total of 23 eligible case-control studies recruiting 4,598 participants were enrolled. Our results suggested that elevated CRP levels in both the serum and CSF were prominently in PD patients. Besides, an unite analysis for peripheral blood and CSF strengthens the outcomes. Subgroup analyses displayed that irrespective of CRP or hs-CRP being the measurement maker, the CRP levels in peripheral blood significantly increased in PD patients comparing with the controls. In addition, sensitivity analysis appeared to indicate that the results were stable. Nevertheless, funnel plot with slight asymmetry and Egger's test $(P<0.001)$ did detect the occurrence of publication bias because of the four studies $(23,28,31,33)$. Study by Liu et al. (23) recruited the smallest sample size of only 40 participants and PD patients with the longest duration. The patients and controls enrolled in research by Han et al. (33) were the youngest groups with age no more than 55 years old. Those two studies both reported extremely large effect, and results of Gao et al. (28) and Wang et al. (31) also exerted quite an influence on overall effect size. Notwithstanding, those four studies did not change the general results in sensitivity analysis; the publication bias may be due to the unpublished negative studies and the existence of the high heterogeneity among the studies. In all, the results indicated an association of higher CRP levels and the risk of PD. However, 


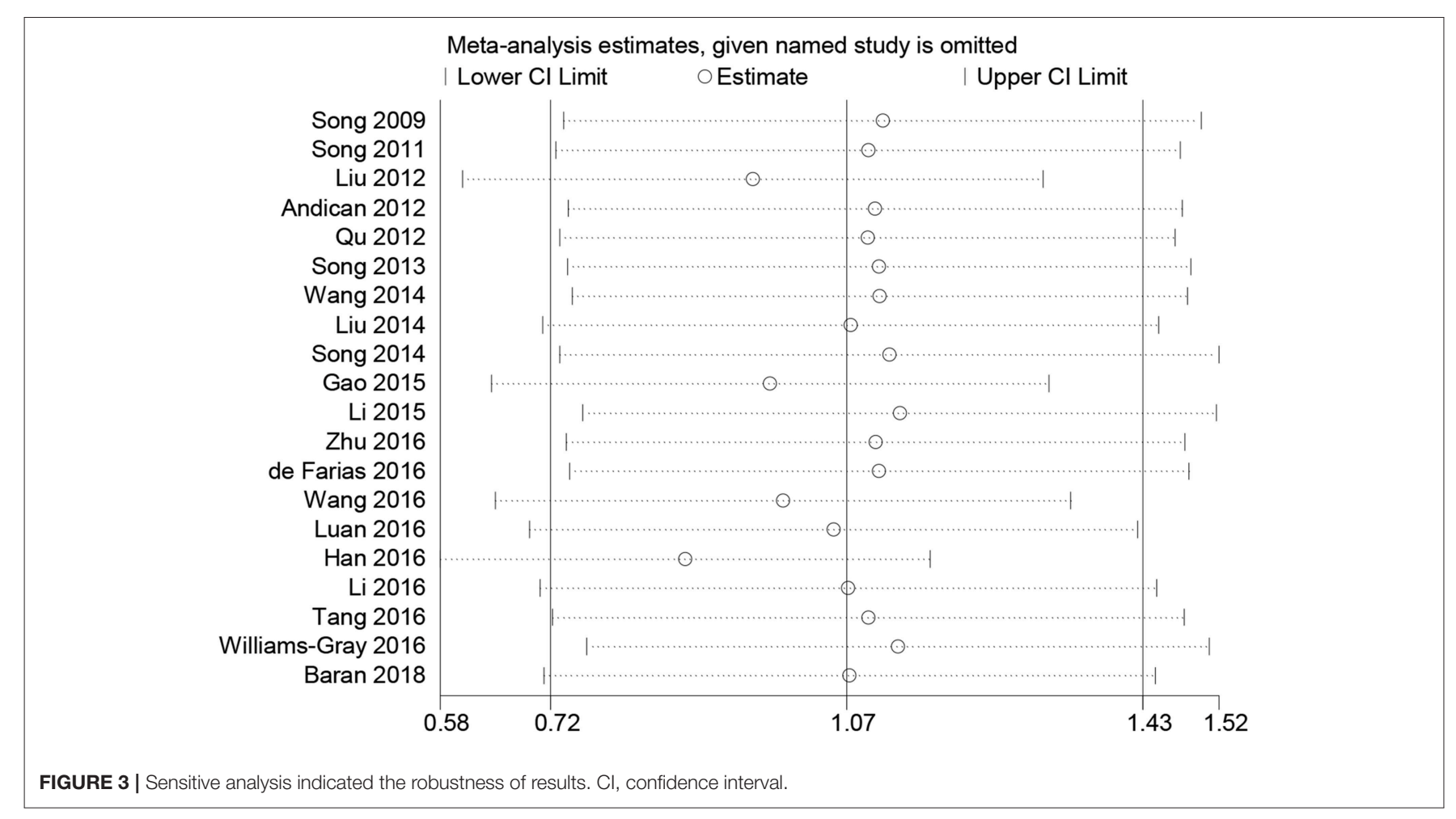

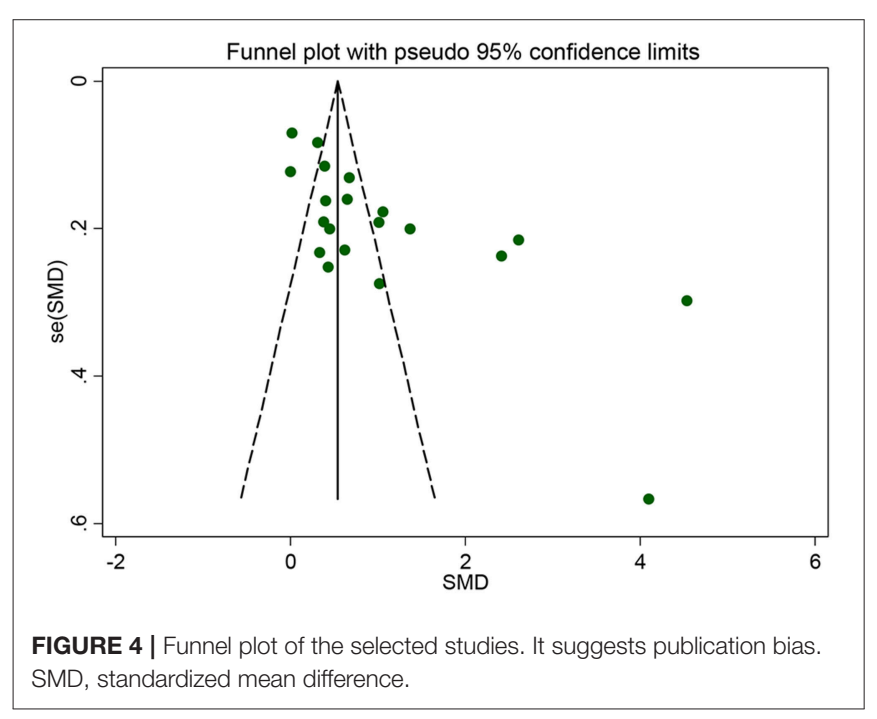

further large scale and well-design trials are warranted to verify our conclusion.

The results of this review only suggested a correlation between CRP levels and PD, but could not completely delineate whether inflammation plays a causal role in $\mathrm{PD}$, or if $\mathrm{PD}$ leads to inflammatory processes. Furthermore, some other confounding factors might influence CRP levels in PD patients. There was considerable variation in the data of disease duration in the included studies, suggesting the probable existence of some confounders (e.g., anti-PD drugs, comorbidity). Some studies
$(15,35)$ measured the difference of hs-CRP level in blood that was not statistically significant between PD patients and controls, when the patients were treated with drugs like levodopa, dopamine receptor agonists and so on. Besides, Andican et al. (15) also found no correlation between the hs-CRP level in plasma and the daily dosage of levodopa or the duration of PD. In addition, de Farias et al. (17) recommended that the anti-PD drugs might increase the inflammatory state. Due to the rarely relative studies included, we could not do a further analysis of the confounding factors such as anti-PD drugs and comorbidity which might be had some affects to our conclusion. Moreover, in a cross-sectional study (46), the authors did not find any correlation between plasma CRP levels and disease duration, levodopa dose, depression, psychosis, dementia, or cognitive decline. However, Lindqvist et al. (47) reported a strong correlation between high CRP levels in CSF and disease duration, fatigue and depression and dementia. Thus, further studies are needed to verify an association between confounding factors such as levodopa treatment or comorbidity in PD and CRP levels.

In present, the mechanisms of elevated CRP levels and neuroinflammation underlying the pathophysiology of PD are still not completely elucidated. As a neurodegenerative disorder, $\mathrm{PD}$ is associated with progressive dopaminergic neuronal degeneration in the substantia nigra. And its prominent neuropathological feature is the presence of Lewy bodies predominantly composed of fibrillar $\alpha$ synuclein (1). At the cellular level, aggregated $\alpha$-synuclein can promote microglial activation and stimulate the secretion of inflammatory molecules, evoking neuroinflammation. In turn, neuroinflammation may trigger cascade of deleterious 


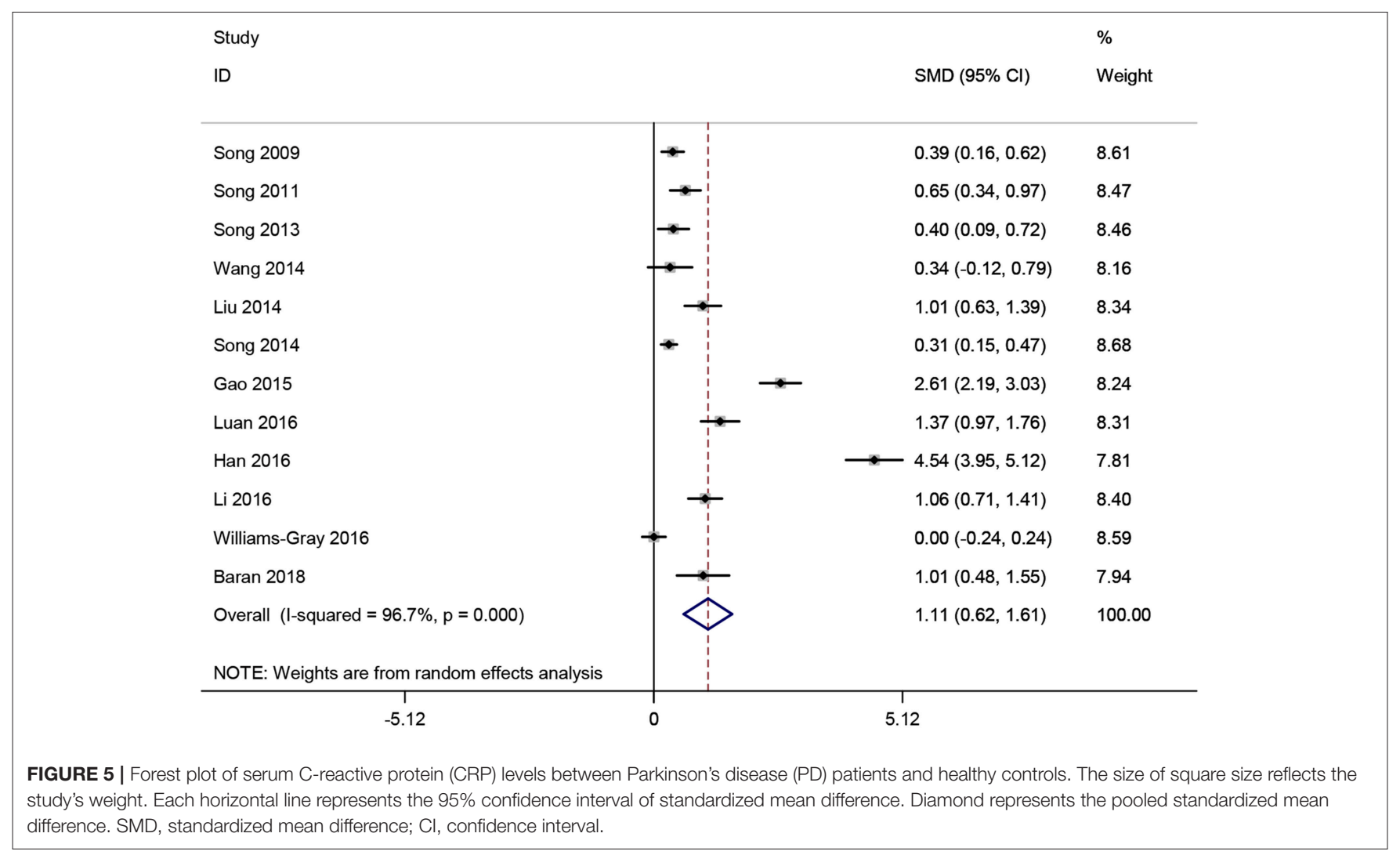

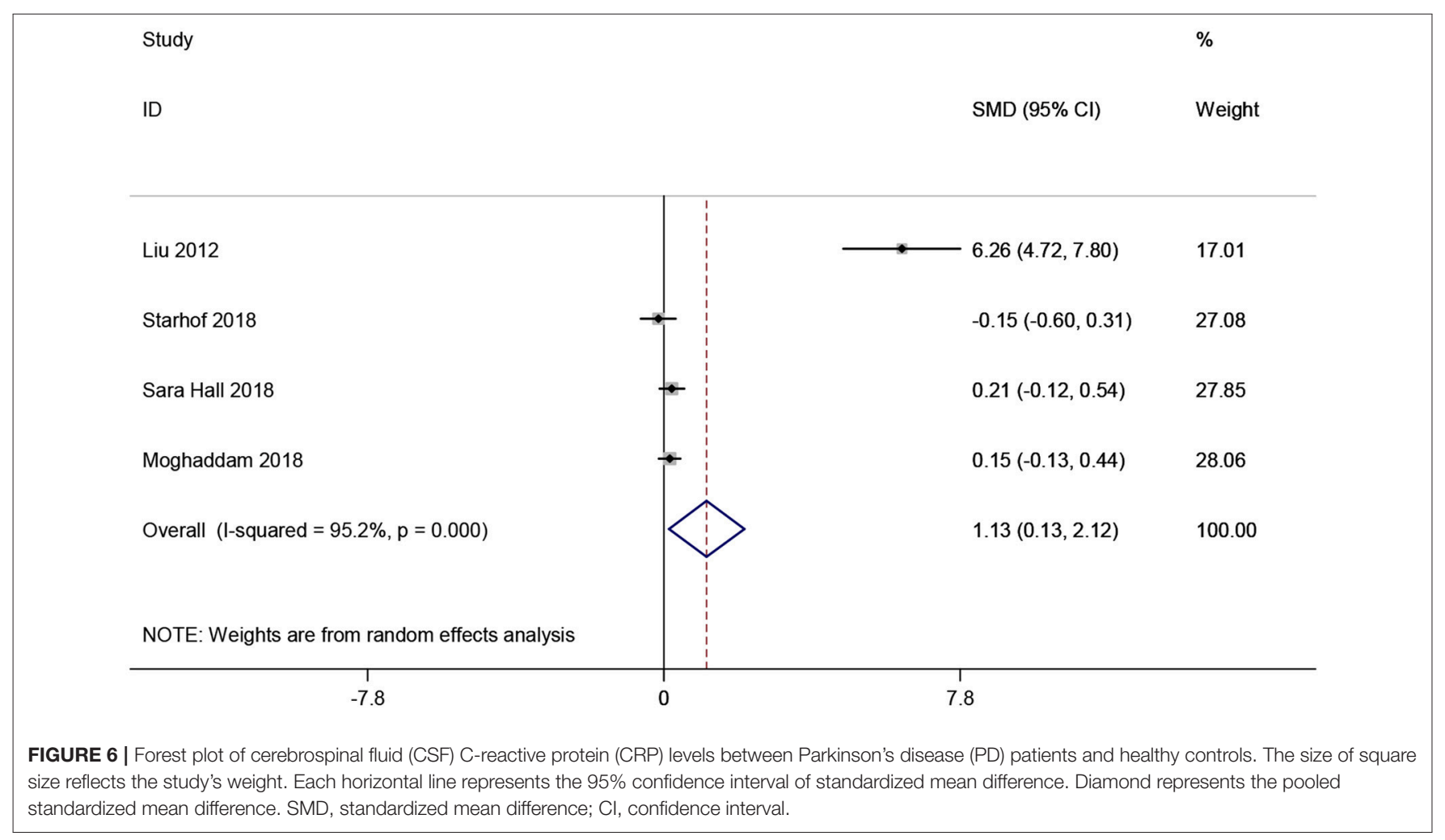


events, such as oxidative stress and cytokine-receptormediated apoptosis, thereby exacerbating dopaminergic neurodegeneration $(5,48)$. Furthermore, epidemiological studies observed that long-term taking anti-inflammatory medications could delay or prevent dopaminergic cell death through inhibiting the pro-inflammatory responses of microglia (11). Simultaneously, as one the most important bio-maker of inflammation, CRP could also be generated by neurons and microglia in the central nervous system according to the post-mortem studies on patients with $\mathrm{AD}$ or intracerebral hemorrhage, or animal studies (49-51). Taken together, there is growing evidence that support an association between neuroinflammation and the initiation and progression of PD pathophysiology.

As we know, this is the most comprehensive meta-analysis of all the published studies that focused on the CRP levels in peripheral blood and CSF between PD patients and matched controls. Though a previous review conducted by Qin (52) assessed several peripheral inflammatory cytokine levels in PD, they only included data from six studies for analyzing CRP levels in PD patients. In this review, a total of 4,598 participants from 23 studies were recruited to estimate the risk, which largely enhanced the reliability of the results. In addition, we focused on CRP levels not only in peripheral blood, but also CSF, which might reflect inflammatory process both of periphery and central nervous system. Our results indicated a significant higher CRP levels in both blood and CSF in patients suffering PD compared with controls. What's more, most of the included studies were of high quality, which further supported that inflammatory cytokine CRP might be involved in the pathological mechanism of PD.

\section{REFERENCES}

1. Kalia LV, Lang AE. Parkinson's disease. Lancet. (2015) 386:896-912. doi: 10.1016/S0140-6736(14)61393-3

2. Chaudhuri KR, Schapira AH. Non-motor symptoms of Parkinson's disease: dopaminergic pathophysiology and treatment. Lancet Neurol. (2009) 8:46474. doi: 10.1016/S1474-4422(09)70068-7

3. Ascherio APSM. The epidemiology of Parkinson's disease: risk factors and prevention. Lancet Neurol. (2016) 15:1257-72. doi: 10.1016/S1474-4422(16)30230-7

4. Tansey MG, Goldberg MS. Neuroinflammation in Parkinson's disease: its role in neuronal death and implications for therapeutic intervention. Neurobiol. Dis. (2010) 37:510-8. doi: 10.1016/j.nbd.2009.11.004

5. Hirsch EC, Hunot S. Neuroinflammation in Parkinson's disease: a target for neuroprotection? Lancet Neurol. (2009) 8:382-97. doi: 10.1016/S1474-4422(09)70062-6

6. McGeer PL, Itagaki S, Boyes BE, McGeer EG. Reactive microglia are positive for HLA-DR in the substantia nigra of Parkinson's and Alzheimer's disease brains. Neurology. (1988) 38:1285-91 doi: 10.1212/WNL.38.8.1285

7. McGeer PL, McGeer EG. Inflammation and neurodegeneration in Parkinson's disease. Parkinsonism Relat Disord. (2004) 10(Suppl. 1):S3-7. doi: 10.1016/j.parkreldis.2004.01.005

8. Chen H, Zhang SM, Hernan MA, Schwarzschild MA, Willett WC, Colditz GA, et al. Nonsteroidal anti-inflammatory drugs and the risk of Parkinson disease. Arch Neurol. (2003) 60:1059-64. doi: 10.1001/archneur.60. 8.1059

9. Bassani TB, Vital MA, Rauh LK. Neuroinflammation in the pathophysiology of Parkinson's disease and therapeutic evidence of anti-inflammatory drugs. Arq Neuropsiquiatr. (2015) 73:616-23. doi: 10.1590/0004-282X201 50057
There are still some limitations that should be carefully interpreted. Firstly, though we performed subgroup analyses to reduce the source of heterogeneity, there were remaining substantial heterogeneity, implying that some confounding variables might exit. Secondly, some studies were not included in this meta-analysis as they assessed the median absolute values of CRP in PD patients and healthy controls and data from these studies were unable to extract for analyses (53-56). Thirdly, the exist of publication bias may also affect the results of this review.

\section{CONCLUSION}

In summary, the current systematic review reveals the CRP levels of blood and CSF were significantly elevated in PD patients when compared with healthy controls, indicating that CRP might be a risk factor for PD or PD leads to an inflammatory response.

\section{AUTHOR CONTRIBUTIONS}

$\mathrm{XQ}$ and YX designed the study, reviewed the literature, conducted the statistical analysis and drafted of the manuscript collectively. JWu, LG, and YH performed summary tables, edited pictures, and discussed on the manuscript. JWa contributed significantly to the study design and critically revised the final manuscript.

\section{FUNDING}

This work was supported by the National Natural Science Foundation of China (Nos. 81460181 and 81801268).

10. Manthripragada AD, Schernhammer ES, Qiu J, Friis S, Wermuth L, Olsen JH, et al. Non-steroidal anti-inflammatory drug use and the risk of Parkinson's disease. Neuroepidemiology. (2011) 36:155-61. doi: 10.1159/000325653

11. Rees K, Stowe R, Patel S, Ives N, Breen K, Clarke CE, et al. Non-steroidal anti-inflammatory drugs as disease-modifying agents for Parkinson's disease: evidence from observational studies. Cochrane Database Syst Rev. (2011) 9:11. doi: 10.1002/14651858.CD008454.pub2

12. Gabay C, Kushner I. Acute-phase proteins and other systemic responses to inflammation. $N$ Engl J Med. (1999) 340:448-54. doi: 10.1056/NEJM199902113400607

13. Pepys MB, Baltz ML. Acute phase proteins with special reference to C-reactive protein and related proteins (pentaxins) and serum amyloid A protein. $A d v$ Immunol. (1983) 34:141-212. doi: 10.1016/S0065-2776(08)60379-X

14. Luan YY, Yao YM. The clinical significance and potential role of C-reactive protein in chronic inflammatory and neurodegenerative diseases. Front Immunol. (2018) 9:1302. doi: 10.3389/fimmu.2018.01302

15. Andican G, Konukoglu D, Bozluolcay M, Bayulkem K, Firtiina S, Burcak G. Plasma oxidative and inflammatory markers in patients with idiopathic Parkinson's disease. Acta Neurol Belg. (2012) 112:155-9. doi: 10.1007/s13760-012-0015-3

16. Song IU, Cho HJ, Kim JS, Park IS, Lee KS. Serum hs-CRP levels are increased in de Novo Parkinson's disease independently from age of onset. Eur Neurol. (2014) 72:285-9. doi: 10.1159/000363570

17. de Farias CC, Maes M, Bonifacio KL, Bortolasci CC, de Souza NA, Brinholi FF, et al. Highly specific changes in antioxidant levels and lipid peroxidation in Parkinson's disease and its progression: disease and staging biomarkers and new drug targets. Neurosci Lett. (2016) 617:66-71. doi: 10.1016/j.neulet.2016.02.011

18. Williams-Gray CH, Wijeyekoon R, Yarnall AJ, Lawson RA, Breen DP, Evans JR, et al. Serum immune markers and disease progression in an incident 
Parkinson's disease cohort (ICICLE-PD). Mov Disord. (2016) 31:995-1003. doi: $10.1002 / \mathrm{mds} .26563$

19. Hall S, Janelidze S, Surova Y, Widner H, Zetterberg H, Hansson O. Cerebrospinal fluid concentrations of inflammatory markers in Parkinson's disease and atypical parkinsonian disorders. Sci Rep. (2018) 8:13276. doi: 10.1038/s41598-018-31517-z

20. Liberati A, Altman DG, Tetzlaff J, Mulrow C, Gøtzsche PC, Ioannidis JPA, et al. The PRISMA statement for reporting systematic reviews and metaanalyses of studies that evaluate healthcare interventions: explanation and elaboration. BMJ. (2009) 339:b2700. doi: 10.1136/bmj.b2700

21. Song IU, Kim JS, Chung SW, Lee KS. Is there an association between the level of high-sensitivity C-reactive protein and idiopathic Parkinson's disease? A comparison of Parkinson's disease patients, disease controls and healthy individuals. Eur Neurol. (2009) 62:99-104. doi: 10.1159/000222780

22. Song IU, Chung SW, Kim JS, Lee KS. Association between high-sensitivity Creactive protein and risk of early idiopathic Parkinson's disease. Neurol Sci. (2011) 32:31-4. doi: 10.1007/s10072-010-0335-0

23. Liu J, Yu NW, Li XJ, Sun HB, Yang YS. Changes of humoral immune function in patients with Parkinson disease. J. Clin. Neurol. (2012) 25:293-5.

24. Qu Y. Study on the Association Between C-Reactive Protein Levels in Plasma and Parkinson's Disease. Dalian Medical University (2012). p. 32.

25. Song IU, Kim YD, Cho HJ, Chung SW. Is neuroinflammation involved in the development of dementia in patients with Parkinson's disease? Intern Med. (2013) 52:1787-92 doi: 10.2169/internalmedicine.52.0474

26. Wang XL. Non-motor Symptoms in Patients With Parkinson's Disease Correlations With TNF $\alpha, I L-6, s I L-2 R$ and hsCRP in Serum. Dalian Medical University (2014). p. 41.

27. Liu Y, Zhang Q, Hong Y, Wen HP, Wu SY. Clinical study of anklebrachial index and high-sensitivity C-reactive protein in elderly patients with parkinson's disease and mild cognitive impairment. Guangxi Med J. (2014) 1237-9. doi: 10.11675/j.issn.0253-4304.2014.09.15

28. Gao XR, Li N, Xue YD, Shi N. Correlation between the peripheral blood and the depressive and anxiety symptoms of patients suffering from Parkinson's disease. J Int Psychiatry. (2015) 42:60-3. doi: 10.13479/j.cnki.jip.2015.06.020

29. Li GH. Risk factors and clinical features and polymorphism of ATP13A2 gene in patients with Parkinson's disease in Xinjiang. Xinjiang Medical University (2015) 144.

30. Zhu XJ, Han XJ, Jin LL, Li HX, Wang CJ. Correlation between levels of UA, HsCRP and HCY in Parkinson's disease and distribution of traditional Chinese medicine syndromes. J Sichuan Trad Chin Med. (2016) 34:66-8.

31. Wang XM, Zhang YG, Li AL, Long ZH, Wang D, Li XX, et al. Relationship between levels of inflammatory cytokines in the peripheral blood and the severity of depression and anxiety in patients with Parkinson's disease. Eur Rev Med Pharmacol Sci. (2016) 20:3853-6.

32. Luan L, Xu YC, Jiang XL. Relationship between IL-6, CRP levels and hallucinations in PD patients. J Clin Exp Med. (2016) 15:2356-9. doi: $10.3969 / j . i s s n .1671-4695.2016 .23 .026$

33. Han X, Wang BJ, Huang YQ, Zhang YF, Lei Q. Correlation analysis of anxiety, depression, sleep and fatigue in patients with Parkinson's disease. $J$ Int Psychiatry. (2016) 43:594-6. doi: 10.13479/j.cnki.jip.2016.04.006

34. Li ZJ, Zou W, Yang Y. Correlation of serum inflammatory factors with depression in patients with Parkindon's disease. Acta Med Univ Sci Technol Huazhong. (2016) 45:593-6.

35. Tang L, Tong YS, Yang XL. Study of correlation between plasma hypersensitive C-reactive protein and Parkinson's disease. XinJiang Med J. (2016) 46:806-8.

36. Starhof C, Winge K, Heegaard NHH, Skogstrand K, Friis S, Hejl A. Cerebrospinal fluid pro-inflammatory cytokines differentiate parkinsonian syndromes. J Neuroinflamm. (2018) 15:305. doi: 10.1186/s12974-018-1339-6

37. Baran A, Bulut M, Kaya MC, Demirpence O, Sevim B, Akil E, et al. High-sensitivity C-reactive protein and high mobility group box-1 levels in Parkinson's disease. Neurol Sci. (2018) 40:167-73. doi: $10.1007 / \mathrm{s} 10072-018-3611-z$

38. Sanjari Moghaddam H, Valitabar Z, Ashraf-Ganjouei A, Mojtahed Zadeh M, Ghazi Sherbaf F, Aarabi MH. Cerebrospinal fluid C-reactive protein in Parkinson's disease: associations with motor and non-motor symptoms. Neuromol Med. (2018) 20:376-85. doi: 10.1007/s12017-018-8499-5

39. Stang A. Critical evaluation of the Newcastle-Ottawa scale for the assessment of the quality of nonrandomized studies in meta-analyses. Eur J Epidemiol. (2010) 25:603-5. doi: 10.1007/s10654-010-9491-z
40. Hedges LV, Pigott TD. The power of statistical tests in meta-analysis. Psychol Methods. (2001) 6:203-17. doi: 10.1037/1082-989X.6.3.203

41. Higgins JPT, Green S. Cochrane Handbook for Systematic Reviews of Interventions. The Cochrane Collaboration (2011). 12:15p.

42. Higgins JP, Thompson SG. Quantifying heterogeneity in a meta-analysis. Stat Med. (2002) 21:1539-58. doi: 10.1002/sim.1186

43. Egger M, Davey SG, Schneider M, Minder C. Bias in meta-analysis detected by a simple, graphical test. BMJ. (1997) 315:629-34. doi: $10.1136 / \mathrm{bmj} .315 .7109 .629$

44. Lau J, Ioannidis JP, Schmid CH. Quantitative synthesis in systematic reviews. Ann Intern Med. (1997) 127:820-6. doi: 10.7326/0003-4819-127-9-199711010-00008

45. Mantel N, Haenszel W. Statistical aspects of the analysis of data from retrospective studies of disease. J Natl Cancer Inst. (1959) 22:719-48.

46. Hassin-Baer S, Cohen OS, Vakil E, Molshazki N, Sela BA, Nitsan Z, et al. Is C-reactive protein level a marker of advanced motor and neuropsychiatric complications in Parkinson's disease? J Neural Transm. (2011) 118:539-43. doi: 10.1007/s00702-010-0535-Z

47. Lindqvist D, Hall S, Surova Y, Nielsen HM, Janelidze S, Brundin L, et al. Cerebrospinal fluid inflammatory markers in Parkinson's disease-Associations with depression, fatigue, and cognitive impairment. Brain Behav Immun. (2013) 33:183-9. doi: 10.1016/j.bbi.2013.07.007

48. Codolo G, Plotegher N, Pozzobon T, Brucale M, Tessari I, Bubacco $\mathrm{L}$, et al. Triggering of inflammasome by aggregated alpha-synuclein, an inflammatory response in synucleinopathies. PLoS ONE. (2013) 8:e55375. doi: 10.1371/journal.pone.0055375

49. Di Napoli M, Godoy DA, Campi V, Masotti L, Smith CJ, Parry JA, et al. C-reactive protein in intracerebral hemorrhage: time course, tissue localization, and prognosis. Neurology. (2012) 79:690-9. doi: 10.1212/WNL.0b013e318264e3be

50. Yasojima K, Schwab C, McGeer EG, McGeer PL. Human neurons generate C-reactive protein and amyloid P: upregulation in Alzheimer's disease. Brain Res. (2000) 887:80-9 doi: 10.1016/S0006-8993(00)02970-X

51. Juma WM, Lira A, Marzuk A, Marzuk Z, Hakim AM, Thompson CS. C-reactive protein expression in a rodent model of chronic cerebral hypoperfusion. Brain Res. (2011) 1414:85-93. doi: 10.1016/j.brainres.2011.07.047

52. Qin XY, Zhang SP, Cao C, Loh YP, Cheng Y. Aberrations in peripheral inflammatory cytokine levels in Parkinson disease: a systematic review and meta-analysis. JAMA Neurol. (2016) 73:1316-24. doi: 10.1001/jamaneurol.2016.2742

53. Shu Y, Li R, Qiu W, Chang Y, Sun X, Fang L, et al. Association of serum gamma-glutamyltransferase and C-reactive proteins with neuromyelitis optica and multiple sclerosis. Mult Scler Relat Disord. (2017) 18:65-70. doi: 10.1016/j.msard.2017.09.021

54. Umemura A, Oeda T, Yamamoto K, Tomita S, Kohsaka M, Park $\mathrm{K}$, et al. Baseline plasma C-reactive protein concentrations and motor prognosis in Parkinson disease. PLoS ONE. (2015) 10:e136722. doi: 10.1371 /journal.pone. 0136722

55. Lindqvist D, Kaufman E, Brundin L, Hall S, Surova Y, Hansson O. Non-motor symptoms in patients with Parkinson's disease - correlations with inflammatory cytokines in serum. PLoS ONE. (2012) 7:e47387. doi: 10.1371/journal.pone.0047387

56. Sawada H, Oeda T, Umemura A, Tomita S, Hayashi R, Kohsaka M, et al. Subclinical elevation of plasma C-reactive protein and illusions/hallucinations in subjects with Parkinson's disease: case-control study. PLoS ONE. (2014) 9:e85886. doi: 10.1371/journal.pone.0085886

Conflict of Interest Statement: The authors declare that the research was conducted in the absence of any commercial or financial relationships that could be construed as a potential conflict of interest.

Copyright $\odot 2019$ Qiu, Xiao, Wu, Gan, Huang and Wang. This is an open-access article distributed under the terms of the Creative Commons Attribution License (CC $B Y)$. The use, distribution or reproduction in other forums is permitted, provided the original author(s) and the copyright owner(s) are credited and that the original publication in this journal is cited, in accordance with accepted academic practice. No use, distribution or reproduction is permitted which does not comply with these terms. 